\title{
Evidence for a connection between photospheric and wind structure in HD 64760^
}

\author{
A. Kaufer ${ }^{1}$, R. K. Prinja ${ }^{2}$, and O. Stahl ${ }^{3}$ \\ 1 European Southern Observatory, Alonso de Cordova 3107, Casilla 19001, Santiago 19, Chile \\ 2 Department of Physics \& Astronomy, University College London, Gower Street, London WC1E 6BT, UK \\ 3 Landessternwarte Heidelberg, Königstuhl 12, 69117 Heidelberg, Germany
}

Received 2 August 2001 / Accepted 16 November 2001

\begin{abstract}
We report on the results of an extended optical spectroscopic monitoring campaign on the earlytype B supergiant HD64760 (B0.5 Ib). The study is based on high-resolution echelle spectra obtained with the Landessternwarte Heidelberg's Heros instrument at ESO La Silla. Ninety-nine spectra were collected over 103 nights between January 19 and May 1, 1996. The H $\alpha$ line shows a characteristic profile with a central photospheric absorption superimposed by symmetrically blue- and red-shifted wind-emission humps. The time-averaged line profile is well described by a differentially rotating and expanding radiation-driven wind: the redistribution of the wind emission flux into a double peak profile is interpreted in terms of the resonance zone effect in rotating winds as first described by Petrenz \& Puls (1996). Detailed time-series analyses of the line profile variations across the $\mathrm{H} \alpha$ profile reveal for the first time in an optical data set of HD 64760 a periodic 2.4-day modulation of the inner and outer flanks of the $\mathrm{H} \alpha$ emission humps. The stronger modulations of the inner flanks of the emission humps at photospheric velocities are due to complex width variations of the underlying photospheric H $\alpha$ profile. The weaker variations of the outer flanks are in phase and reflect variations at the base of the stellar wind. The detected 2.4-day modulation period together with a second period of 1.2 days (in the red emission hump only) is in excellent agreement with the outer-wind modulation periods as reported by Fullerton et al. (1997) from intensive IUE UV time-series observations in 1993 and 1995. The 2.4-day period is further detected in the photospheric He I $\lambda 4026$ line as prograde traveling (pseudo-)absorption and emission features. The observed variability pattern is indicative for low-order non-radial pulsations in the photosphere of HD 64760. The non-radial pulsations are identified as the source of persistent, regularly spaced stellar surface structure which is maintained throughout the photosphere - wind transition zone (this work) out into the UV regime of the terminal velocity outflow.
\end{abstract}

Key words. stars: early-type - stars: individual (HD 64760) - stars: mass loss - stars: rotation - stars: oscillation

\section{Introduction}

Recent optical and ultraviolet (UV) spectroscopic monitoring campaigns have revealed systematic, patterned variability in the stellar winds of luminous hot stars on time scales associated with processes on the stellar surface (cf. for O stars e.g. Prinja et al. 1995, 2001; Massa et al. 1995; Howarth et al. 1995; for late B- and early A-supergiants Kaufer et al. 1996a, 1996b and for early Bhypergiants Rivinius et al. 1997). One of the key questions for understanding the nature and origin of this wind variability remains its connection to the stellar photosphere.

HD 64760 is an intrinsically fast rotating less luminous early-type B supergiant (B0.5 Ib). It is particularly wellsuited for spatial-structure studies: because of the high

Send offprint requests to: A. Kaufer, e-mail: akaufer@eso.org

* Based on observations collected at the European Southern Observatory at La Silla, Chile (Proposal ID 56.D-0235). observed $v \sin i$ of $265 \mathrm{~km} \mathrm{~s}^{-1}$ (cf. below) it is very likely viewed almost equator-on which maximizes the effects of stellar rotational modulation. Indeed, HD 64760 exhibits the most striking and clearly systematic wind structure of all $\mathrm{O}$ and early B supergiant stars studied in the UV (Prinja et al. 1995; Massa et al. 1995). With the discovery of strictly periodic and sinusoidal modulations of the SiIII, Sirv, and NV UV resonance lines with periods of 1.2 and 2.4 days (Fullerton et al. 1997). HD 64760 has become a key object in the study of spatially structured hot star winds and their connection to the stellar surface.

The detected wind-modulation periods with approximately a half and a quarter of an estimated rotational period of $P_{\text {rot }}=4.8$ days is interpreted as a broad twoand four-armed corotating circumstellar structure which modulates the optical depth of the outer stellar wind of HD 64760. Cranmer \& Owocki (1996) developed a consistent hydrodynamic model of corotating interaction 


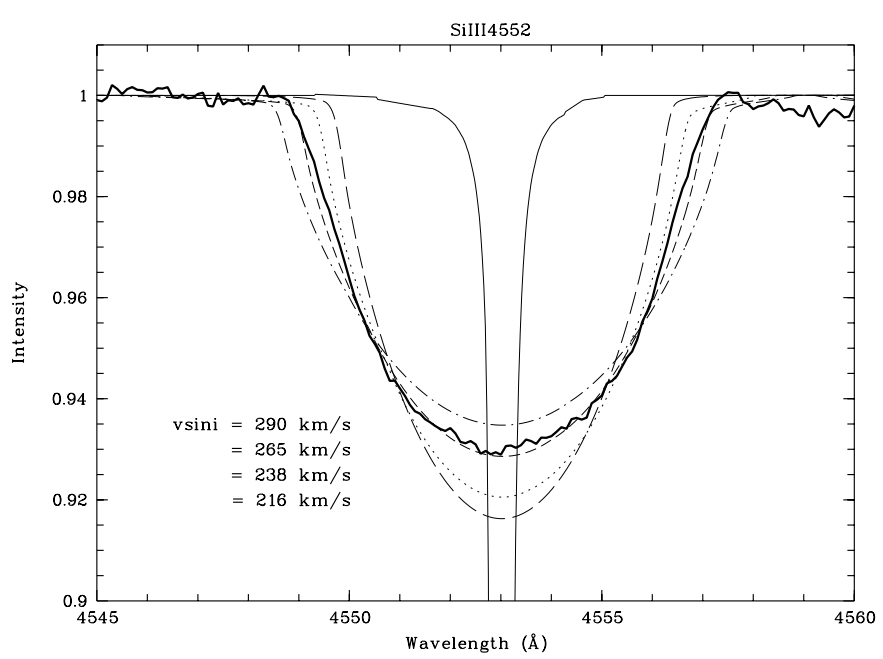

Fig. 1. The observed mean Si III $\lambda 4552$ line profile (thick solid line) in comparison with rotationally broadened synthetic profiles for $v \sin i=216 \mathrm{~km} \mathrm{~s}^{-1}$ (long dashed), $238 \mathrm{~km} \mathrm{~s}^{-1}$ (dotted), $265 \mathrm{~km} \mathrm{~s}^{-1}$ (dashed), and $290 \mathrm{~km} \mathrm{~s}^{-1}$ (dash-dotted). After rotational broadening, the synthetic spectra have been convolved with an Gaussian instrumental profile corresponding to a resolving power of $R=20000$. The thin solid line shows the synthetic profile for zero rotation.

regions (CIRs) caused by the collision of fast and slow wind streams which are rooted in large-scale perturbations at the base of the wind. In the case of HD 64760 a regular and longitudinally-spaced two- and four-sector geometry on the stellar surface seems most applicable. Baade (1984) detected for the first time photospheric lineprofile variations in highest-quality optical spectra of the Si III $\lambda 4552$ and HeI $\lambda 6678$ lines which he interpreted as high $(|m|$ large) and low order $(|m| \approx 2)$ non-radial pulsations (NRPs) with respective periods of $\sim 0.1$ and $\sim 0.5$ days. Howarth et al. (1998) could detect the 1.2-day period in the UV photospheric lines of the IUE MEGA data set with a weak signature for a prograde traveling NRP pattern in the corresponding phase diagrams. Such photospheric low order NRP patterns might provide the large-scale perturbations at the base of the wind needed to build large-scale wind structure. Unfortunately the UV lines of HD 64760 as observed intensively with IUE did not sample the transition zone between the photosphere and the base of the stellar wind. This missing link can be filled observationally with optical long-term spectroscopic time series as presented for the first time for HD 64760 in this work.

There is some evidence in other OB stars that perturbations in the photosphere propagate into the stellar wind. Reid \& Howarth (1996) have detected a $8.5 \mathrm{hr}$ period in the optical photospheric and $\mathrm{H} \alpha$ line profiles of the $\zeta$ Puppis O4I(n)f. For the O7.5 III star $\xi$ Persei wind variability with a period of 2 days could be traced down to near-photospheric velocities (cf. de Jong et al. 2001 and references therein).

\section{Observations}

Our optical spectroscopic data set of HD 64760 was obtained about one year after the 1995 IUE MEGA campaign. In 1996 the fiber-linked echelle spectrograph HEROS (Stahl et al. 1996) was mounted for an extended 4-month monitoring campaign at the ESO 50-cm telescope at La Silla. In the 103 nights between January 19 and May 1 (MJD 50101-50204) a total of 99 spectra of HD 64760 were obtained with a typical sampling of one spectrum per night. The two arms of the HEROs spectrograph cover simultaneously the spectral range from $3450 \AA$ to $5560 \AA$ in the blue channel and from $5820 \AA$ to $8620 \AA$ in the red channel with a resolving power of 20000 . The $S / N$-ratio of the spectra strongly depends on the wavelength and is lowest in the near-UV spectral range. A typical $S / N>150$ was obtained in the red spectral range with an exposure time of $40 \mathrm{~min}$.

Flatfield and wavelength-calibration exposures have been obtained with the instrument-internal Halogen and Thorium-Argon lamps, the latter not more than two hours apart from the science exposure. All spectra have been reduced semi-automatically with ESO-MIDAS using a modified version of the standard echelle package (Stahl et al. 1993).

\section{The time-averaged spectrum}

The large number of individual spectra obtained during the observing campaign allows us to compose in a first step a high-quality mean spectrum of HD 64760. For this purpose the 99 normalized spectra were averaged with weights according to the achieved $S / N$-ratios.

\subsection{The projected equatorial rotation velocity}

The high quality of the mean spectrum allows us first to determine the projected equatorial rotation velocity $v \sin i$ with good accuracy. The Si III $\lambda 4552$ line was selected for the fitting of rotationally broadened synthetic profiles because it is the least blended, non-Balmer photospheric line in the spectral range of Heros. As will be shown later in this work, no significant line profile variations could be detected in the Si III lines of our time series with $S / N$-ratios of $\sim 100$ per individual spectrum in contrast to the line profile variability found by Baade (1984) on short time scales and highest $S / N$ spectra. Therefore, to the level of the achieved spectral quality no significant additional broadening due to the averaging process of the time series spectra has to be expected. A Doppler-broadened synthetic Si III $\lambda 4552$ absorption line was computed for the stellar parameters given in Table 1 using the Bнт LTE line formation code (Baschek et al. 1966); the depth of the line was scaled to match exactly the measured equivalent width $\left(W_{\lambda}=410 \mathrm{~m} \AA\right)$ of the observed Si III $\lambda 4552$ line. This profile was then convolved with theoretical rotation profiles of different $v \sin i$ using the rotation profile as described by Gray (1992) with a limb-darkening 


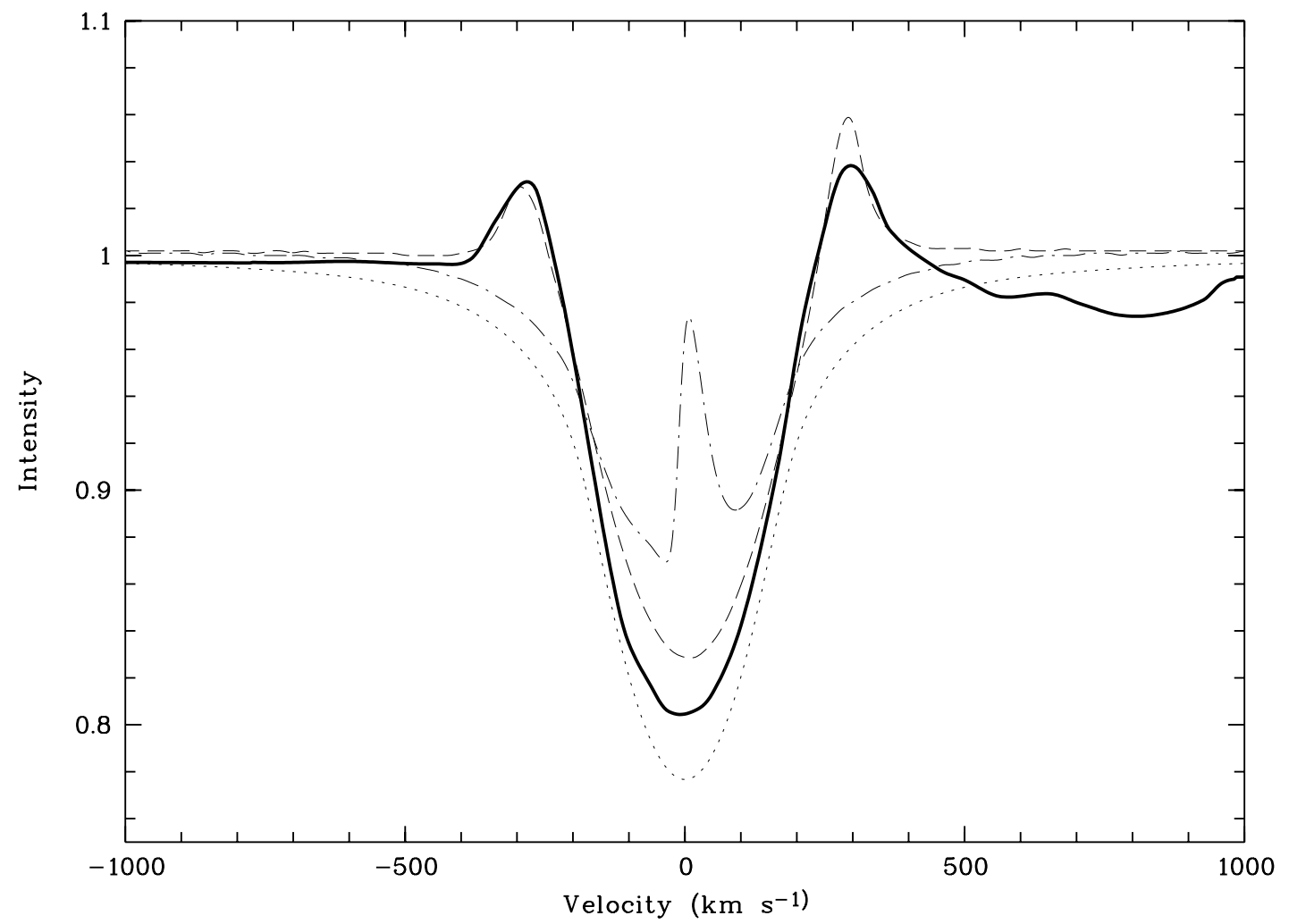

Fig. 2. $\mathrm{H} \alpha$ profile: thick solid line: $S / N$-ratio weighted average of time series (telluric absorption lines removed), dotted line: rotationally broadened photospheric model profile, dash-dotted line: spherical symmetric, non-rotating wind model $(\dot{M}=$ $\left.9.0 \times 10^{-7} M_{\odot} \mathrm{yr}^{-1}\right)$, dashed line: rotating wind model $\left(\dot{M}=9.0 \times 10^{-7} M_{\odot} \mathrm{yr}^{-1}, v_{\mathrm{eq}}=290 \mathrm{~km} \mathrm{~s}^{-1}, \sin i=1\right)$. The broad absorption feature at $+800 \mathrm{~km} \mathrm{~s}^{-1}$ is caused by the photospheric $\mathrm{C}$ II absorption blend.

parameter $\varepsilon=0.55$. For the instrumental profile of Heros a Gaussian profile with a width corresponding to a resolving power of $R=20000$ was convolved with the rotationally broadened line profiles. Figure 1 shows the results for the four different values $v \sin i$ discussed in this work in comparison with the observed Si III $\lambda 4552$ line. A best fit is obtained for $v \sin i=265 \pm 5 \mathrm{~km} \mathrm{~s}^{-1}$ which will be used throughout this work. The value is clearly higher than the $v \sin i$ values used in previous works. Massa et al. (1995) had adopted the value of $v \sin i=238 \mathrm{~km} \mathrm{~s}^{-1}$ from Hoffleit \& Jaschek (1982) of which the origin is difficult to trace. Even lower values of $v \sin i$ have been derived by Bernacca \& Perinotto (1970) $\left(v \sin i=220 \mathrm{~km} \mathrm{~s}^{-1}\right)$ and by Howarth et al. (1998) $\left(v \sin i=216 \mathrm{~km} \mathrm{~s}^{-1}\right)$, the latter determined from UV photospheric lines. These low $v \sin i$ values are clearly not compatible with our observed optical line profiles as can be seen in Fig. 1. Our new determination of $v \sin i=265 \mathrm{~km} \mathrm{~s}^{-1}$ translates into a smaller upper limit for the stellar rotation period of HD 64760 of $P_{\text {rot }} / \sin i=4.2$ days.

\subsection{The mean $\mathrm{H \alpha}$ line profile}

Figure 2 shows the time-averaged $\mathrm{H} \alpha$ line (thick solid line) together with different synthetic line profiles (cf. below). The $\mathrm{H} \alpha$ profile displays a characteristic double-peak structure with a blueward and a redward shifted emission hump and a central absorption. The latter is well centered on the rest wavelength of $\mathrm{H} \alpha$. The red emission hump is slightly stronger than the blue; on its outer red edge the red emission wing is overlaid by the broad C II blend at $+800 \mathrm{~km} \mathrm{~s}^{-1}$. Throughout this paper we use a systemic velocity of $v_{\text {sys }}=+18 \mathrm{~km} \mathrm{~s}^{-1}$ as previously used for HD 64760 and which is consistent with our spectra. All velocities have been transformed by this value and therefore are given relative to the laboratory wavelengths. The position of the blue and red emission humps are measured to be $-290 \mathrm{~km} \mathrm{~s}^{-1}$ and $+300 \mathrm{~km} \mathrm{~s}^{-1}$, respectively. Since the two emission peaks are strongly asymmetric the velocity of the maximum intensity is given here. The $\mathrm{H} \alpha$ emissionline intensity is weak and reaches a value of about $4 \%$ above the stellar continuum in the two emission peaks. This points towards a moderate mass-loss rate as expected for a Ib supergiant. For a more quantitative estimate of the photospheric contribution to the $\mathrm{H} \alpha$ wind profile, we computed synthetic Balmer line profiles for $\mathrm{H} \alpha$ to $\mathrm{H} \gamma$ using ATLAs 9 LTE model atmospheres and the BALMER 9 LTE line formation code (Kurucz 1993). We adopted an effective temperature of $T_{\text {eff }}=25000 \mathrm{~K}$ according to a spectral type of B0.5 Ib using the temperature scale by Crowther (1999). For solar abundances and a microturbulence of $\xi_{\text {micro }}=2 \mathrm{~km} \mathrm{~s}^{-1}$ we obtained a good fit for the mostly unblended $\mathrm{H} \beta$ line for a gravity of $\log g(\mathrm{cgs})=3.3$. The characteristic double emission as seen in $\mathrm{H} \alpha$ is still 


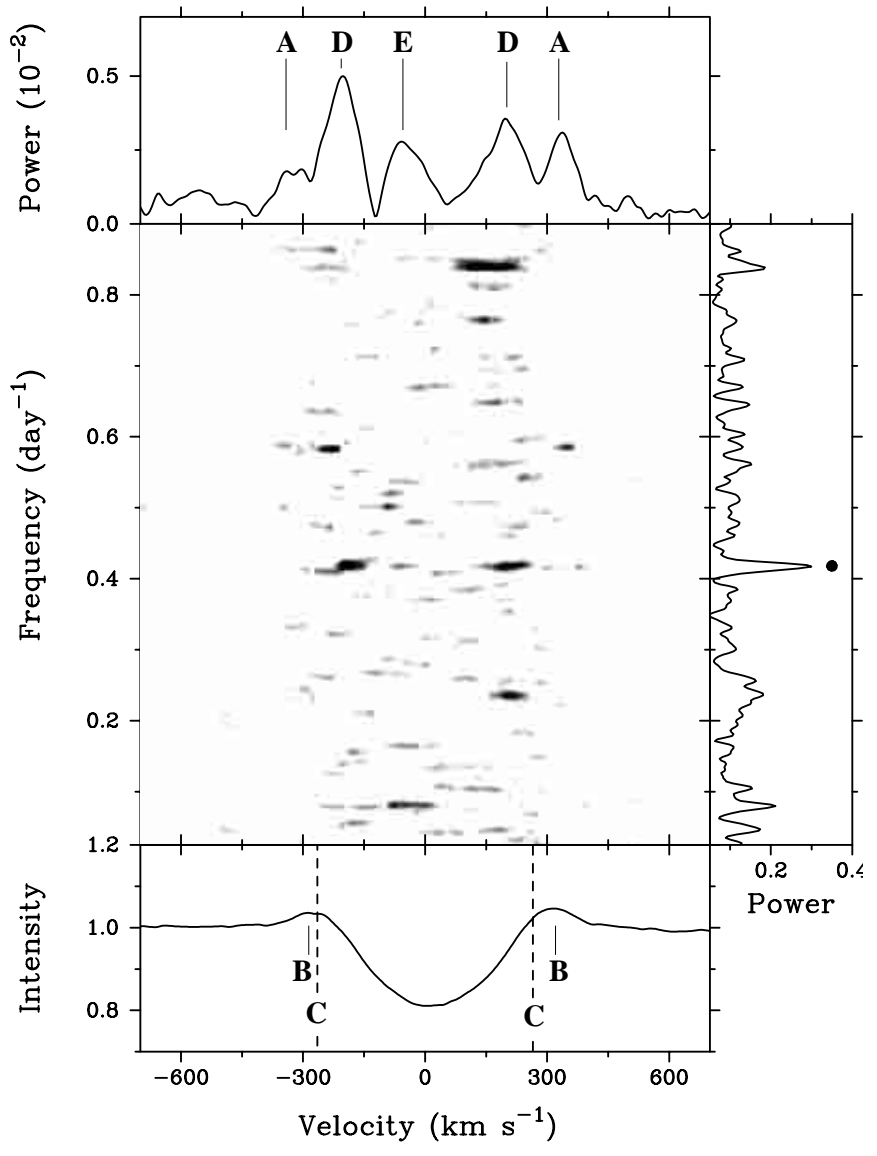

Fig. 3. Time series analysis of the $\mathrm{H} \alpha$ line. Top: power distribution for the frequency of $0.42 \mathrm{day}^{-1}$ corresponding to a period of 2.4 days. Center: Cleaned discrete Fourier transform spectrum of the $\mathrm{H} \alpha$ line. Bottom: mean $\mathrm{H} \alpha$ profile, the vertical dashed lines indicate $\pm v \sin i$. Right: power distribution averaged over the displayed velocity interval of the line as function of frequency. The strongest peak with the frequency of 0.42 day $^{-1}$ is indicated by a dot. In the top and bottom panel the spectral and power spectrum features $\mathrm{A}-\mathrm{E}$ as listed in Table 2 are indicated.

discernable in our mean spectrum for $\mathrm{H} \beta$ if the residuals with respect to the synthetic Balmer line profile are inspected.

Table 1 summarizes the stellar parameters used and derived in this work.

The comparison of the synthetic absorption profile (Fig. 2, dotted line) with the observed $\mathrm{H} \alpha$ profile (thick solid line) confirms the strong contribution of the underlying photospheric absorption profile. If we subtract the photospheric model profile from the observed $\mathrm{H} \alpha$ profile we are left with two Gaussian-shaped emission peaks. The positions of the blue and red emission humps were determined with a Gaussian fit to $-260 \mathrm{kms}^{-1}$ and $+275 \mathrm{~km} \mathrm{~s}^{-1}$ - still quite symmetric about zero-velocity but shifted towards slightly lower absolute velocities if compared to the positions of maximum intensity.

HD 64760 is an intrinsically fast rotating supergiant with a high probability to be seen equator-on.
Table 1. Stellar parameters for HD 64760.

\begin{tabular}{lcl}
\hline Parameter & Value & Reference \\
\hline Spectral Type & B0.5 Ib & Hoffleit \& Jaschek (1982) \\
$V$ & 4.24 & Hoffleit \& Jaschek (1982) \\
$(B-V)$ & -0.15 & Hoffleit \& Jaschek (1982) \\
$v \sin i\left(\mathrm{~km} \mathrm{~s}^{-1}\right)$ & 265 & this work \\
$v_{\infty}\left(\mathrm{km} \mathrm{s}^{-1}\right)$ & 1500 & Massa et al. (1995) \\
$T_{\text {eff }}(\mathrm{K})$ & 25000 & B0.5 Ib: Crowther (1999) \\
$R_{\star} / R_{\odot}$ & 22 & Humphreys \& McElroy (1984) \\
$M_{\star} / M_{\odot}$ & 20 & Schaller et al. (1992) \\
$\dot{M} / M_{\odot} \mathrm{yr}^{-1}$ & $9.0 \times 10^{-7}$ & this work \\
$P_{\text {rot }} / \sin i($ days $)$ & 4.2 & this work \\
\hline
\end{tabular}

Consequently, the high ratio between equatorial rotation velocity and terminal wind velocity of $v_{\text {eq }} / v_{\infty}=$ $265 / 1500=0.18$ already suggests that the differential rotation of its expanding radiation-driven wind must play a major rôle in the formation of the characteristic doublepeaked $\mathrm{H} \alpha$ wind profile.

Petrenz \& Puls (1996) have described in detail the effects of the differential rotation of an expanding envelope on the $\mathrm{H} \alpha$ profile. A key to the understanding of the effects of rotation plus expansion is the so-called resonance zone effect: the differential rotation of the expanding envelope introduces a twist of the resonance zones (i.e., the zones where the radially projected speed of the wind material equals the Doppler displacement with respect to the rest wavelength of the line) near the stellar surface, i.e., the photosphere - wind transition zone. Near the line center, the optical depth is decreased due to a twist of the resonance zone away from the star, but on the other hand increased for blue and red velocities due to a twist towards the star (cf. Petrenz \& Puls 1996, their Fig. 6). With the strong $\rho^{2}$ density dependence of $\mathrm{H} \alpha$ line formation, the rotationally twisted resonance zones result in a redistribution of emission line flux from the line center towards red and blue emission humps near $\pm v \sin i$.

To demonstrate the presence of the resonance zone effect in the $\mathrm{H} \alpha$ profile of HD 64760 we have implemented the model described by Petrenz \& Puls (1996) for a differentially rotating wind with the simplified assumption of a spherically symmetric density stratification throughout the wind (in fact, in a recent work Petrenz \& Puls (2000) derive from self-consistent $2 \mathrm{D}$ rotating wind models an oblate wind morphology for fast rotating winds of hot stars but demonstrate that the therewith determined mass-loss rates do not deviate more than $20 \%$ from the ones determined with previous 1D models). The dashed line in Fig. 2 shows the computed line profile for a stellar effective temperature of $T_{\text {eff }}=25000 \mathrm{~K}$, a stellar radius of $R_{\star}=22 R_{\odot}$, a terminal wind velocity of $v_{\infty}=1500 \mathrm{~km} \mathrm{~s}^{-1}$, a wind initial velocity of $v_{0}=10 \mathrm{~km} \mathrm{~s}^{-1}$, a wind acceleration parameter $\beta=0.8$, a mass-loss rate of $\dot{M}=9.0 \times 10^{-7} M_{\odot} \mathrm{yr}^{-1}$, and an equatorial rotation velocity of $v_{\mathrm{eq}}=290 \mathrm{~km} \mathrm{~s}^{-1}$ (inclination of $\sin i=1$ ). As underlying photospheric profile, the synthetic Balmer model profile as describe above was used. The only two parameter which were adjusted 
to match the observed profile were the mass-loss rate and the rotation velocity. For comparison, Fig. 2 shows with a dashed-dotted line the same wind model without rotation, i.e., $v_{\text {eq }}=0 \mathrm{~km} \mathrm{~s}^{-1}$ (but the same rotationally broadened underlying photospheric profile). For the estimate of the best fit parameters, emphasis was given to the two emission humps. The position and width of the humps are well fitted, while the intensity ratio of the red to blue emission is higher than observed. We will show in the following section that in particular the two $\mathrm{H} \alpha$ emission peaks are highly variable. Our analysis will show that this variability points to the existence of co-rotating, non spherical-symmetric large scale structures in the $\mathrm{H} \alpha$ lineforming wind region for which a static wind model cannot be approriate. Therefore, our simple differentially rotating wind model with a spherical symmetric density stratification cannot be expected to model the mean $\mathrm{H} \alpha$ profile in great detail. The quality of the fit for the central absorption is rather poor. This is not surprising since the use of static LTE model atmospheres and line formation is definitely not appropriate for a B0.5 Ib supergiant - especially for the Doppler core of the $\mathrm{H} \alpha$ line NLTE effects have to be expected. Our photospheric model fits all Balmer lines apart from $\mathrm{H} \alpha$ reasonably well, which indicates a very sudden onset of NLTE effects for the core of the $\mathrm{H} \alpha$ profile. The equatorial rotation velocity used for the rotating wind model $\left(v_{\mathrm{eq}} \sin i=290 \mathrm{~km} \mathrm{~s}^{-1}\right)$ is higher than the measured value for $v \sin i$ of $265 \mathrm{~km} \mathrm{~s}^{-1}$. However, since we have to date no detailed understanding of the innermost acceleration zone of a wind starting from a rotating stellar surface, a straightforward interpretation of this discrepancy is difficult to provide.

\section{Time series analysis}

Our Heros time-series data set of HD 64760 permits the study of the line profile variability in the Balmer and He I lines. The variations are strongest and clearly detectable for the $\mathrm{H} \alpha$ line but close to the detection limit for all remaining lines, the latter due to the small amplitudes of the variations $(\sigma(\mathrm{H} \alpha)<1.5 \%)$ and the moderate to low $S / N$-ratio particularly in the blue spectral region.

\subsection{The $H_{\alpha}$ line}

Time-series analyses of the $\mathrm{H} \alpha$ line-profile variations have been performed in the velocity - time domain using the discrete Fourier transform (DFT) methods as described e.g. by Gies \& Kullavanijaya (1988) and Baade (1988). An iterative CLEAN algorithm (Roberts et al. 1987) was used to deconvolve the window function from the discrete Fourier transform (200 iterations, gain 0.2). The ClEANed discrete Fourier transforms (CDFT) are presented in 2D (velocity-frequency) spectra with the ClEANed power coded in gray scales.

Figure 3 shows in the central panel the CDFT of $\mathrm{H} \alpha$ for the frequency domain of $0.0-0.9$ day $^{-1}$. A clear detection of systematic power over the line profile is found

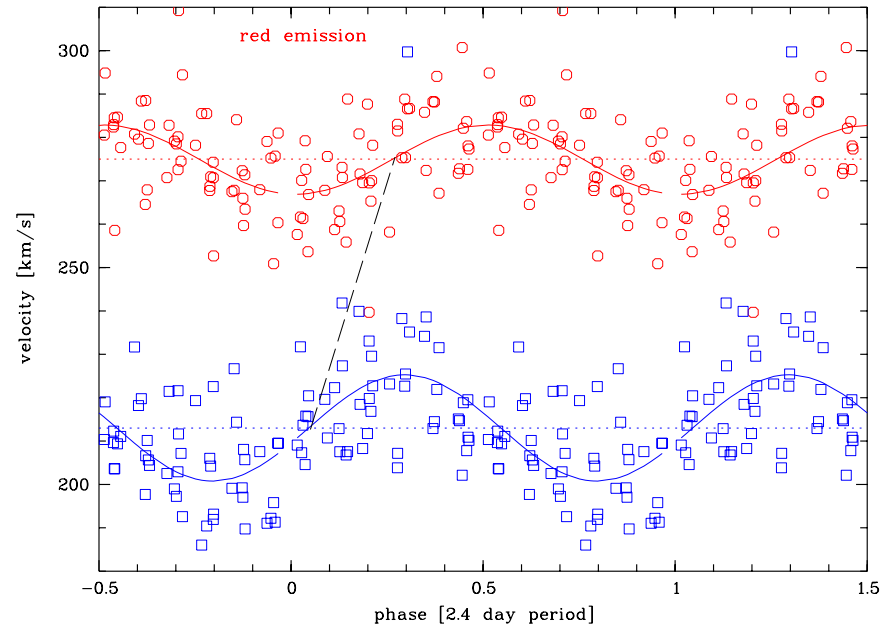

Fig. 4. Variations of the $\mathrm{H} \alpha$ emission-hump positions in velocities for a period of 2.4 days (circles: red emission hump, squares: blue emission hump). The measured blue velocities have been offset by $+475 \mathrm{~km} \mathrm{~s}^{-1}$ for better display only. The semi-amplitudes of the red and blue sine-fit are 8 and $9 \mathrm{~km} \mathrm{~s}^{-1}$, the mean velocities are 275 and $-262 \mathrm{~km} \mathrm{~s}^{-1}$ (dotted lines), and the phase offsets are 0.25 and 0.05 , respectively. The phase lag of 0.2 is indicated with a dashed line. The observed apparent position variability of the red and blue emission peaks is induced by asymmetric modulations of the inner and outer flanks of both emission peaks (cf. text and Fig. 5).

at a frequency of 0.42 day $^{-1}$ which corresponds to a period of 2.4 days. The power is primarily distributed into two power peaks symmetrically placed about zero velocity at velocities of $-200 \mathrm{~km} \mathrm{~s}^{-1}$ and $+200 \mathrm{~km} \mathrm{~s}^{-1}$. A further strong detection is found at half the frequency corresponding to a 1.2-day period but at $+200 \mathrm{~km} \mathrm{~s}^{-1}$ only. Since no power is found on the corresponding blue side, the detection in the red is most likely real and not a left-over from the ClEANing of the window function. On the other hand with the 1-day sampling of our data set we cannot draw any strong conclusions on the significance of the 1.2-day period. Therefore, we will not discuss this period in the following.

The detection of the 2.4 (and possibly 1.2) day periods in our optical dataset is the first independent confirmation of the wind-modulation periods of HD 64760 as determined from the IUE MEGA campaign UV data sets (Fullerton et al. 1997). The simple fact that the modulation periods are found at (near-)photospheric velocities of $\pm 200 \mathrm{~km} \mathrm{~s}^{-1}$ suggests that the prominent wind modulation as probed by the UV resonance lines at high outflow velocities is rooted to the base of the wind, i.e., the photosphere - wind transition zone. The identification of the photospheric source of the 2.4-day variability and its possible coupling to the lower wind regions will be subject of the following analysis and discussion.

A closer look at the power distribution over the $\mathrm{H} \alpha$ line around the frequency of 0.42 day $^{-1}$ is provided in Fig. 3, top panel. For this purpose the CDFT spectrum was summed over a frequency interval of \pm 0.01 day $^{-1}$ and 


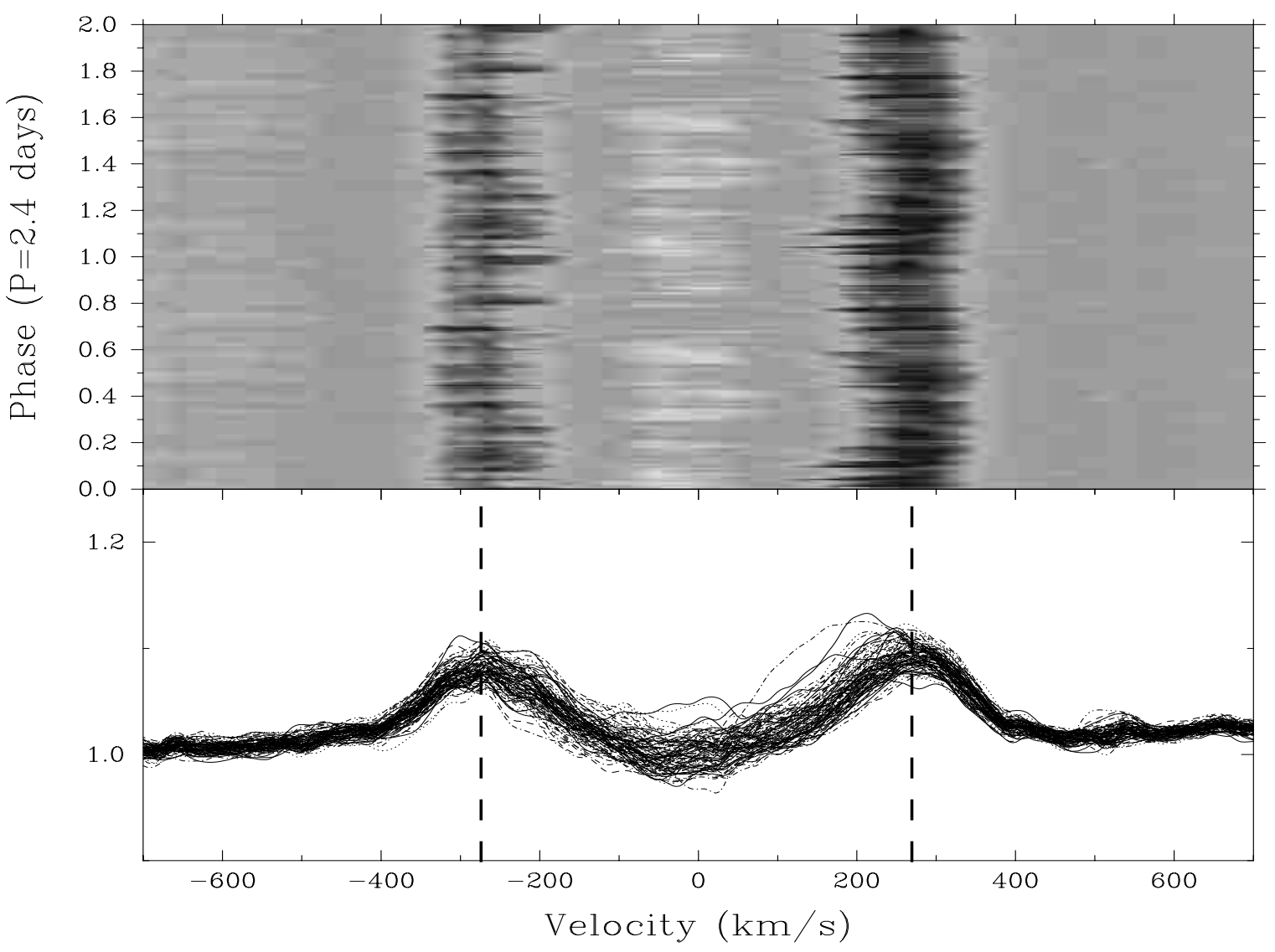

Fig. 5. Dynamic phase spectrum of the $\mathrm{H} \alpha$ line for a period of 2.4 days (top panel). The phase spectrum was constructed from the residual spectra (bottom panel) with respect to the photospheric model spectrum as shown in Fig. 2 (dotted line). Grey scale coding is black: emission - grey: continuum - white: absorption. The values for $\pm v \sin i$ are indicated by vertical dashed lines in the bottom panel. The larger modulation amplitude of the inner flanks of the emission humps is clearly seen at photospheric velocities $\left(\left|v_{\mathrm{r}}\right|<v \sin i\right)$ while the weaker modulations of the outer flanks are observed at base-of-the-wind velocities $\left(\left|v_{\mathrm{r}}\right|>v \sin i\right)$.

is plotted as function of the line-of-sight velocity. Five distinct peaks in the power distribution are centered at velocities of (i) -200 and $+200 \mathrm{~km} \mathrm{~s}^{-1}$, (ii) -320 and $+335 \mathrm{~km} \mathrm{~s}^{-1}$, and (iii) $-50 \mathrm{~km} \mathrm{~s}^{-1}$. As reference the average $\mathrm{H} \alpha$ profile is shown in the bottom panel of Fig. 3 together with indications of the $\pm v \sin i$ velocities.

Table 2 compiles the above-described spectral and 2.4-day period power-spectrum features of the $\mathrm{H} \alpha$ line. If sorted by their radial velocity, the features show a high degree of symmetry about the the line center - pairs of features are marked by the capital letters A to D in Table 2.

The fact that the line profile variability is localized at higher (pair A) and lower (pair D) velocities with respect to the two emission humps (pair B) and $\pm v \sin i$ (pair C) indicates a possible connection of the emission humps on the one hand with the stellar wind variability and on the other hand with the photospheric variability.

Therefore, we will examine in the following the 2.4-day variations of the $\mathrm{H} \alpha$ emission humps in more detail. In a first step the individual $\mathrm{H} \alpha$ profiles of the time series were fitted with a triple-Gaussian profile: two Gaussian emission profiles at the approximate positions of the emission
Table 2. Spectral and power spectrum features of the $\mathrm{H} \alpha$ line sorted by their radial velocity (see also Fig. 3).

\begin{tabular}{l|rc}
\hline feature & $\begin{array}{r}\text { velocity } \\
{\left[\mathrm{km} \mathrm{s}^{-1}\right]}\end{array}$ & pair ID \\
\hline blue power peak & -320 & $\mathrm{~A}$ \\
blue emission hump & -290 & $\mathrm{~B}$ \\
$-v \sin i$ & -265 & $\mathrm{C}$ \\
blue power peak & -200 & $\mathrm{D}$ \\
central power peak & -50 & $\mathrm{E}$ \\
systemic velocity & 0 & \\
red power peak & +200 & $\mathrm{D}$ \\
$+v \sin i$ & +265 & $\mathrm{C}$ \\
red emission hump & +300 & $\mathrm{~B}$ \\
red power peak & +335 & $\mathrm{~A}$ \\
\hline
\end{tabular}

humps plus a central Gaussian absorption profile fit the observed profiles with very good quality. Out of the 9 initially free fit parameters (height, position, and width for each of the three Gaussians) only the positions of the two (red and blue) Gaussian emission profiles show significant $(3 \sigma)$ variability with the 2.4 day period. Figure 4 shows 
the phase diagram of the measured emission-hump positions for a period of 2.4 days and the zero phase set for the first spectrum of the time series, i.e., for MJD 50100.36. To improve the robustness of the triple-Gaussian fit for the emission-hump position measurements the width of the red and blue Gaussian were fixed to 160 and $180 \mathrm{~km} \mathrm{~s}^{-1}$, respectively, which corresponds to the average width of the emission humps. The measured position variations of the red and blue humps have then been fitted with sine functions as shown in Fig. 4 (the blue measurements and sine-fit have been offset by $+475 \mathrm{~km} \mathrm{~s}^{-1}$ for better display). The semi-amplitudes of the red and blue sinefit are 8 and $9 \mathrm{kms}^{-1}$, the mean velocities are 275 and $-262 \mathrm{~km} \mathrm{~s}^{-1}$, and the phase offsets are 0.25 and 0.05 , respectively. These measurements suggest that periodic variability of the $\mathrm{H} \alpha$ emission-hump positions is responsible for the A and D power spectrum features in the respective flanks of the emission humps. A periodic, purely positionvariable spectral feature is expected to produce in the power spectrum signatures at the positions of the maximum gradient of the line profile, i.e., in our case in the two flanks of the respective emission hump. Further a phase jump of 0.5 is expected between the two power signals of the left and right flank. In Fig. 3 the expected double signature in the flanks of the profile can be identified with the features A and D as well as for the red as for the blue emission - in addition the phase jump between features $\mathrm{A}$ and $\mathrm{D}$ is measured to be of the expected value of 0.5 .

Since position variability could be mimicked by nonsymmetric variations of the shape of the emission humps we inspect in the following directly the variability of the emission-hump profiles. For this purpose we have sorted the 99 spectra of the time series in phase for a period of 2.4 days. Phase zero is set as before for MJD 50100.36. To enhance the contrast of the spectral variations the (constant) photospheric $\mathrm{H} \alpha$ model spectrum as described in Sect. 3 was subtracted from each individual spectrum of the time series. Figure 5 shows the resulting $2 \mathrm{D}$ velocity - phase spectrum over two cycles with the residual intensities coded in grey scales (black: emission - grey: contiuum - white: absorption). Below, all residual spectra from which the dynamic phase spectrum was created are over-plotted.

The strongest line profile variability is seen at -200 and $+200 \mathrm{~km} \mathrm{~s}^{-1}$ in the form of a sinusoidal velocity modulation of the inner flanks of the blue and red emission humps. The amplitude of the modulation is $\sim 40 \mathrm{~km} \mathrm{~s}^{-1}$. At phase $=0.5(0.7)$ the inner flank of the red (blue) emission reaches the highest, at phase $=1.0(1.2)$ the smallest absolute velocity. This periodic "eating" into the emission humps is caused by a periodic variation of the width of the underlying photospheric profile as can be demonstrated by the direct comparison of photospheric line profiles at phase 0.5 and 1.0 , e.g. in the central photospheric absorption of $\mathrm{H} \alpha$. The higher Balmer lines but also the He I $\lambda \lambda 4026,4921$ lines may show the same effect but with much smaller amplitudes for the width broadening. The outer flanks of the emission humps follow the modulation of the inner flanks in phase and sense but with considerably smaller velocity amplitudes, i.e., the flank at $+335 \mathrm{~km} \mathrm{~s}^{-1}$ with an amplitude of $\sim 20 \mathrm{~km} \mathrm{~s}^{-1}$, the flank at $-320 \mathrm{~km} \mathrm{~s}^{-1}$ with an amplitude of less than $\sim 10 \mathrm{~km} \mathrm{~s}^{-1}$. The modulation of the outer flank is localized well beyond $v \sin i$, and therefore must be attributed to variations at the base of the wind. The above described asymmetric modulation of the inner and outer flanks of the red and blue emission humps indeed introduces an apparent variation of the position of the emission features as measured above (Fig. 4). The smaller modulation amplitude of the outer flanks with respect to the inner flanks is reflected in the power distribution with features A being much weaker than features D. Also the variability at $-50 \mathrm{~km} \mathrm{~s}^{-1}$ (feature $\mathrm{E}$ ) is discernible in the dynamic phase spectrum as an $\pm 100 \mathrm{~km} \mathrm{~s}^{-1}$ wide intensity increase near phase 0.75 .

It is important to note that the described line-profile variations do not lead to significant variations in the total equivalent width of the $\mathrm{H} \alpha$ profile. With integration boundaries of $\pm 400 \mathrm{~km} \mathrm{~s}^{-1}$ the equivalent width is measured to $W_{\lambda}=1135 \mathrm{~m} \AA$ with an $\mathrm{rms}$ of $90 \mathrm{~m} \AA$. A period analysis of the equivalent width curve does not show any significant power above a confidence level of $3 \sigma$, especially not at the otherwise prominent frequency of 0.42 day $^{-1}$. This further emphasizes the modulating character of the observed $\mathrm{H} \alpha$ variability.

To summarize: the apparent position variability of the red and blue emission humps of the $\mathrm{H} \alpha$ profile with a period of 2.4 days are caused by asymmetric modulations of the inner and outer flanks of the emission-line profiles with the modulation of the outer flank being weaker than the modulation of the inner flank but both in phase. Between the modulations of the red and blue emissions a phase lag of 0.2 is observed. The modulations of the inner flanks are caused by complex width variations of the underlying photospheric profile while the variations of the outer flanks most likely reflect the induced variations at the base of the wind.

These complex but periodic variations of the photospheric profile underlying $\mathrm{H} \alpha$ are not straightforward to interpret in the context of rotational modulation due to stellar surface structure. Low order non-radial pulsation (NRP) patterns have been favoured by Fullerton et al. (1997) as possible surface structure because of the regularity of the observed UV wind modulations. The marginal detections of NRP characteristics in the photospheric lines of the same data sets by Howarth et al. (1998) gave further support to this interpretation.

The line width variations as found in our optical data set can be interpreted in a simple, purely dynamical, NRP model with horizontal velocity fields of high amplitude: velocity vectors parallel to the stellar surface are best detected at the stellar limbs, i.e., at line-of-sight velocities near $\pm v \sin i$. The observed inner edge variation of the emission humps (feature D) is highly concentrated in velocity space and localized closely to $\pm v \sin i$ (feature $\mathrm{C}$ ) which seems compatible with horizontal pulsation velocity fields. With the state-of-the-art non-radial pulsation 


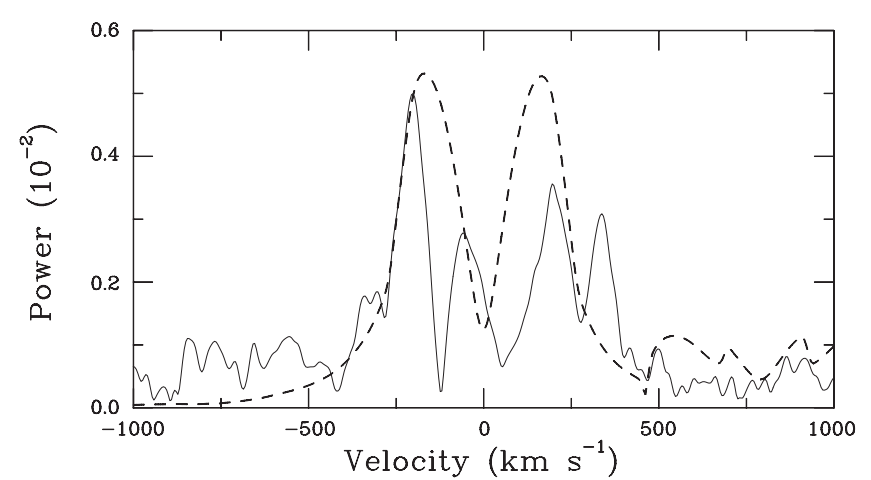

Fig. 6. Power distribution across the $\mathrm{H} \alpha$ line from a $l=1$ $m=1$ non-radial pulsation model (dashed line) in comparison with the observed power distribution (solid line) for a period of 2.4 days.

codes BRUCE and KYLIE by Townsend (1997) it is not possible to produce a power distribution as found in the features D. Even the lowest order $l=|m|=1$ pulsation modes which display the highest-amplitude horizontal velocity fields do not allow to deposit almost all power signal close to the stellar limb rotation velocities (Fig. 6 by R. Townsend, priv. comm.). However, the outer wings of feature D are quite well reproduced by the pulsation model. It is not clear if more sophisticated non-adiabatic pulsation models could provide more physical background for a NRP interpretation of the observed $\mathrm{H} \alpha$ variations of HD 64760. A further possibility to create a more complex power distribution is the presence of multi-periodic non-radial pulsations. The high-quality optical data sets of Baade (1984) which provide very high time resolution of a few hours suggest high and low order NRPs with periods of 0.1 and 0.5 days. Since to date no spectroscopic data set has covered the crucial time scales between a few hours to a few days, a complex (multi-periodic) pulsation spectrum which might result in very complex line profile variations and power spectra cannot be excluded.

The presence of the 2.4-day power in the line-profile variability of $\mathrm{H} \alpha$ at line-of-sight velocities $>v \sin i$, i.e., in the outer flanks of the emission humps (feature A), is of particular interest. Variability in this velocity regime has to be attributed to the variability in the stellar outflow. The simple fact that variability is seen at photospheric and base-of-the-wind velocities with the identical periodicity and LPV signature (i.e., a phase-locked sinusoidal modulation of the emission hump flanks) indicates a direct coupling of photospheric and wind variability. Feature A with absolute velocities larger than $300 \mathrm{~km} \mathrm{~s}^{-1}$ connects our optical data set with the IUE MEGA campaign data sets in velocity space. In the UV the onset of resonance line profile variability could be traced down to outflow velocities of $-240 \mathrm{~km} \mathrm{~s}^{-1}$ in the Si III and Si IV lines, and to $-400 \mathrm{~km} \mathrm{~s}^{-1}$ in the $\mathrm{C}$ IV and N v lines (Fullerton et al. 1997, their Table 3). The finding of the 2.4-day period from photospheric velocities throughout the base and acceleration zone of the wind up to the terminal wind velocity observationally support the presence of persistent regular spatial structures in the atmosphere (=photosphere and expanding envelope) of HD 64760. Therefore, stellar wind structure is rooted in the stellar photosphere - at least for HD 64760. Unfortunately the presented data do not provide deeper insight to the underlying physical mechanism(s) which couple the stellar surface variability to the radiaton-driven outflow to build up such large scale structures.

\subsection{The Hel lines}

The spectral line with the strongest signal in the power spectrum after $\mathrm{H} \alpha$ is the He I $\lambda 4026$ line. We have searched the HERos spectral range for further line profile variability in the same frequency range as for $\mathrm{H} \alpha$ and had a marginal detection for the He I $\lambda 4026$ line at a frequency of 0.42 day $^{-1}$, i.e., the same 2.4 -day period. Figure 7 shows on the bottom panel the mean line profile of the He I $\lambda 4026$ line, above the power distribution and the phase variation of the 2.4-day signal across the line profile. In the top panel the residual spectra with respect to the mean spectrum of the time series have been phase-binned with the 2.4-day period. The resulting 2.4-day phase-binned dynamical spectrum shows a (pseudo-) absorption travelling prograde from $-v \sin i$ at phase 0.0 to $+v \sin i$ at phase 0.5, a (pseudo-) emission feature follows between phase 0.5 and 1.0. Such travelling features are indicative of line profile variations due to photospheric low-order nonradial pulsations (NRP). The weak NRP pattern does not allow a detailed determination of the pulsation mode. On the other hand, the observed NRP pattern with a single (pseudo-) absorption or emission feature visible at all phases is compatible with a $l=-m=2$ mode.

Phases of increased absorption in the He I $\lambda 4026$ line profile coincide with decreased flux in $\mathrm{H} \alpha$. Most prominent at phase 0.5 the absorption feature is located at $+200 \mathrm{~km} \mathrm{~s}^{-1}$ (cf. Fig. 7 , top panel) which coincides in velocity and phase with a decreased flux in the inner flank of the red emission hump of $\mathrm{H} \alpha$ (cf. Fig. 5). This finding further supports the conclusion of Sect. 4.1 that the variability at the inner flanks of the $\mathrm{H} \alpha$ emission humps (pair D) are caused by variations of the underlying photospheric profile.

\section{Discussion}

The extended optical high-resolution time series presented in this work has provided the first ever probe of line-profile variability of HD 64760 in the photosphere and at the base of the wind. This optical data set provides detailed information complementary to the IUE MEGA campaign data sets which revealed systematic large scale structure of the stellar wind beyond 3-5 stellar radii. The $\mathrm{H} \alpha$ line and its $\rho^{2}$ sensitivity provides a powerful resource to investigate the atmosphere structure in the inner few stellar radii. 


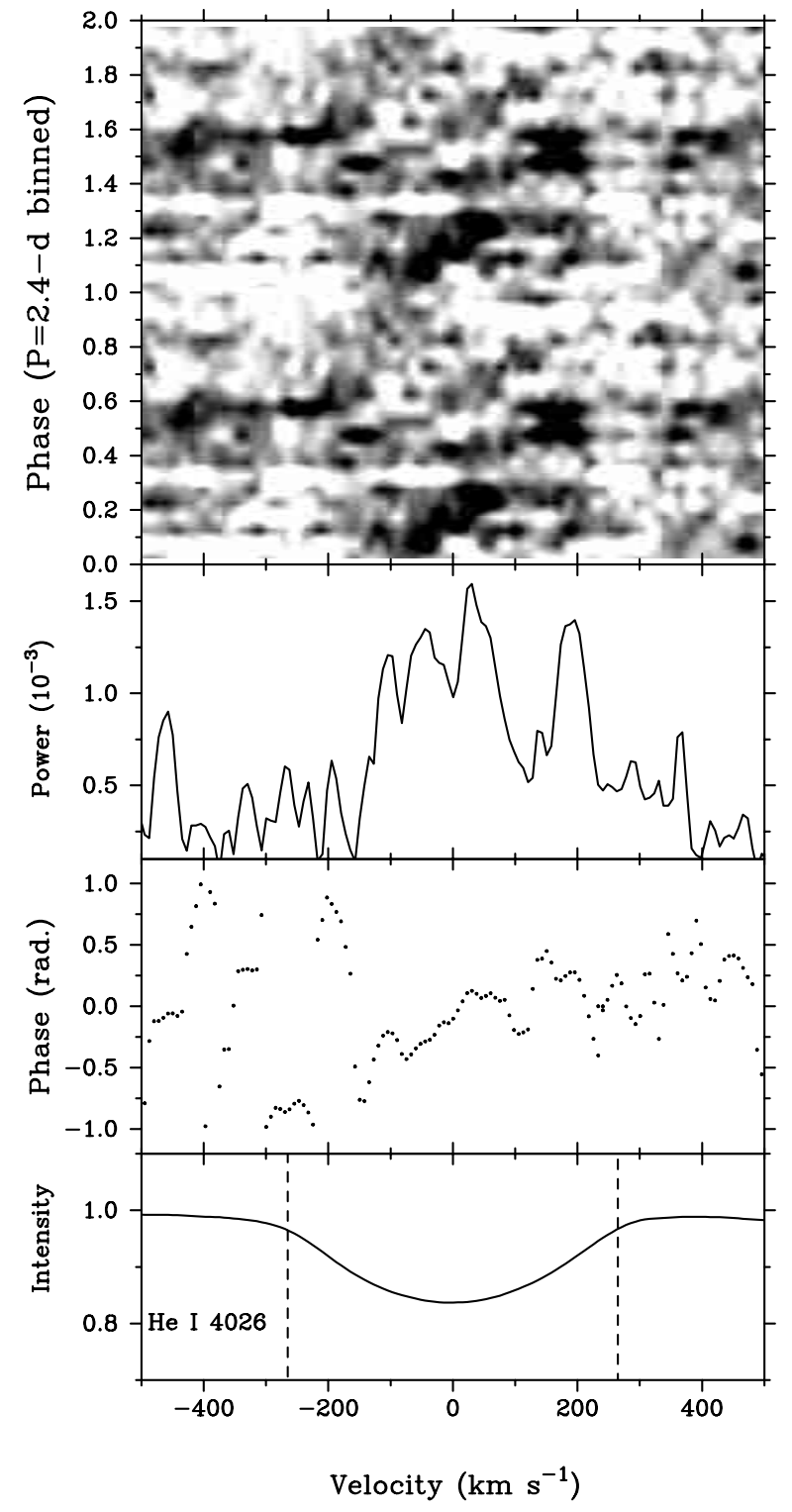

Fig. 7. The He I $\lambda 4026$ line: panels from top to bottom: the 2D phase-binned dynamic spectrum created from the residual spectra with respect to the mean spectrum of the time series, the power distribution across the line profile for the 2.4-day period, the corresponding phase variation of the signal across the line profile, and the mean line profile with vertical lines indicating $\pm v \sin i$.

Our detection of the 2.4-day modulation period in the optical line profile variations of the photosphere - wind transition zone line $\mathrm{H} \alpha$ and the photospheric He I lines provides a crucial link between the star's wind and photosphere structure: large-scale wind structure in HD 64760 is rooted in the stellar photosphere. The 2.4-day period has been stable for at least one year, i.e., the time span between the 1995 IUE and the 1996 Heros campaign. Such a long-term presence of a single modulation period requires a stable clock which is most naturally provided bye the star's own rotation. Our newly determined upper limit for the stellar rotation period of 4.2 days is within the expected errors close to twice the 2.4-day modulation period. If this estimate is correct, the modulating structure on the stellar surface must be also persistent over such a time scale and geometrically divide the stellar surface in two structures with 180 degree azimuthal separation. The most likely sources of such large-scale structure on the stellar surface are either non-radial pulsation patterns or magnetic field structures. From our finding of (pseudo-) absorption and emission features travelling prograde over the He I $\lambda 4026$ line we favour in the case of HD 64760 surface structures due to non-radial low-order $g$-mode pulsations. The NRP pattern in the He I $\lambda 4026$ line is compatible with a $l=-m=2$ pulsation. A NRP in this pulsation mode would structure the stellar surface into four equal quadrants with the respective opposite quadrants in the same physical state - as required for an observed modulation period of half the rotation period.

The gross difference between the observed $\mathrm{H} \alpha$ power distribution at the 2.4-day period (Fig. 3, central panel) and that seen in He I $\lambda 4026$ at the same period (Fig. 7) provides a major constraint for any detailed NRP modeling of HD 64760. Due to its sensitivity to the photosphere - wind transition zone a modeling of $\mathrm{H} \alpha$ variability has to take into account pulsationally induced wind variation effects. Based on the recent works by Townsend (2000a,b) one might speculate here on a possible linkage between low-frequency $g$ modes and wind variability through wave leakage, i.e., the physical link between the features $\mathrm{A}$ in the wind and the features D in the photosphere.

For the small-amplitude variations as found in HD 64760 the quality of our HEROs data set is not sufficient to detect further organized line-profile variability in other photospheric lines or to provide the necessary input for detailed time dependent line-profile modelling. Since the complex variability pattern of the photospheric lines could be an indicator for multi-periodic stellar oscillations, the variability of HD 64760 has to be probed in possible future optical montioring campaigns on the intrinsic stellar pulsation time scales, i.e., $\approx 1$ hour if a pulsation constant of $Q=0.04$ days (Lovy et al. 1984) and the stellar parameters in Table 1 are assumed.

At the same time again the large-scale modulations on the rotation time scale have to be examined in greater detail to e.g. search for near-star signatures of the corotating spiral structures now well-established for the outer wind regions of HD 64760. The innermost formation region of the spiral structure, i.e., the inner collision zone of fast and slow streams in the CIR picture (cf. Cranmer \& Owocki 1996), may well fall into the emission-line forming region of the sensitive $\mathrm{H} \alpha$ line. Because of the rather small geometrical extent close to the star and the rather low density contrast to be expected for the near-to-the-star spiral structures, the transient features possibly introduced in the line profiles can only be detected with highest-quality (i.e., in $S / N$-ratio, spectral, and time resolution) optical time series spectroscopy. 
Acknowledgements. We thank the staff at La Silla Observatory for their kind support for Heros at the ESO 50-cm telescope. We are grateful to our colleague Richard Townsend for the helpful discussions on NRPs and his test model computations. Further we would like to thank Dominik Schäfer and Dugan Witherick for their help in prepartion and handling of the data sets.

\section{References}

Baade, D. 1984, Photospheric velocity fields of early-type supergiants: A preliminary observational report, in Proc. of the 25th Liège International Astrophysical Colloquium, Theoretical Problems in Stellar Stability and Oscillations, Université de Liège, Liège, 115

Baade, D. 1988, in O stars \& Wolf-Rayet Stars, ed. P. S. Conti, \& A. B. Underhill (NASA SP-497, Washington), 137

Bernacca, P. L., \& Perinotto, M. 1970, Contr. Oss. Astrof. Padova in Asiago, 239

Baschek, B., Holweger, H., \& Traving, G. 1966, Abhandl. Hamburger Sternwarte 8, No. 1, 26

Cranmer, S. R., \& Owocki, S. P. 1996, ApJ, 462, 469

Crowther, P. 1999, IAU Symp., 189, 137

de Jong, J. A., Henrichs, H. F., Kaper, L., et al. 2001, A\&A, 368,601

Fullerton, A. W., Massa, D. L., Prinja, R. K., et al. 1997, A\&A, 327,699

Gies, D. R., \& Kullavanijaya, A. 1988, ApJ, 326, 813

Gray, D. F. 1992, The observation and analysis of stellar photospheres, Cambridge Astrophysics Series (Cambridge
University Press, New York)

Hoffleit, D., \& Jaschek, C. 1982, The Bright Star Catalogue (New Haven: Yale Univ. Press)

Howarth, I. D., Prinja, R. K., \& Massa, D. L. 1995, ApJ, 452, L65

Howarth, I. D., Townsend, R. H. D., Clayton, M. J., et al. 1998, MNRAS, 296, 949

Humphreys, R. M., \& McElroy, D. B. 1984, ApJ, 284, 565

Kaufer, A., Stahl, O., Wolf, B., et al. 1996a, A\&A, 305, 887

Kaufer, A., Stahl, O., Wolf, B., et al. 1996b, A\&A, 314, 559

Kurucz, R. 1993, CD-ROM No. 13 (Cambridge, Mass.: SAO)

Lovy, D., Maeder, A., Noels, A., et al. 1984, A\&A, 133, 307

Massa, D. L., Prinja, R. K., \& Fullerton, A. W. 1995, ApJ, 452,842

Petrenz, P., \& Puls, J. 1996, A\&A, 312, 195

Petrenz, P., \& Puls, J. 2000, A\&A, 358, 956

Prinja, R. K., Massa, D. L., \& Fullerton, A. W. 1995, ApJ, 452, L61

Prinja, R. K., Stahl, O., Kaufer, A., et al. 2001, A\&A, 367, 891

Rivinius, T., Stahl, O., Wolf, B., et al. 1997, A\&A, 318, 819

Reid, A. H. N., \& Howarth, I. D. 1996, A\&A, 311, 616

Roberts, D. H., Lehár, J., \& Dreher, J. W. 1987, AJ, 93, 968

Schaller, G., Schaerer, D., Meynet, G., \& Maeder, A. 1992, A\&AS, 96, 269

Stahl, O., Mandel, H., Wolf, B., et al. 1993, A\&AS, 99, 167

Stahl, O., Kaufer, A., Rivinius, T., et al. 1996, A\&A, 312, 539

Townsend, R. H. D. 1997, MNRAS, 284, 239

Townsend, R. H. D. 2000, MNRAS, 318, 1

Townsend, R. H. D. 2000, MNRAS, 319, 289 